\title{
Mystery of Bloom-Gilman duality: A light-front holographic QCD perspective
}

\author{
Aiden B. Sheckler and Gerald A. Miller@* \\ Department of Physics, University of Washington, Seattle, Washington 98195, USA
}

(Received 2 February 2021; accepted 16 April 2021; published 19 May 2021)

\begin{abstract}
Light-front wave functions motivated by holographic constructions are used to study Bloom-Gilman duality, a feature of deep inelastic scattering. Separate expressions for structure functions in terms of quark and hadronic degrees of freedom (involving transition form factors) are presented, with an ultimate goal of obtaining a relationship between the two expressions. A specific two-parton model is defined and resonance transition form factors are computed using previously derived light-front wave functions. A new form of global duality (integral over all values of $x$ between 0 and 1) is derived from the valence quarknumber sum rule. Using a complete set of hadronic states is necessary for this new global duality to be achieved, and the previous original work does not provide such a set. This feature is remedied by amending the model to include a longitudinal confining potential, and the resulting complete set is sufficient to carry out the study of Bloom-Gilman duality. Specific expressions for transition form factors are obtained and all are shown to fall as $1 / Q^{2}$, at asymptotically large values. This is because the Feynman mechanism dominates the asymptotic behavior of the model. These transition form factors are used to assess the validity of the global and local duality sum rules, with the result that both are not satisfied within the given model. Evaluations of the hadronic expression for $q\left(x, Q^{2}\right)$ provide more details about this lack. This result is not a failure of the current model because it shows that the observed validity of both global and local forms of duality for deep inelastic scattering must be related to a feature of QCD that is deeper than completeness. Our simple present model suggests a prediction that Bloom-Gilman duality would not be observed if deep inelastic scattering experiments were to be made on the pion. The underlying origin of the duality phenomenon in deep inelastic scattering is deeply buried within the confinement aspects of QCD, and its origin remains a mystery.
\end{abstract}

DOI: 10.1103/PhysRevD.103.096018

\section{INTRODUCTION}

Two distinct facets of QCD are known. At asymptotically high energies and momentum transfers $Q^{2}$ many hadronic observables can be computed using quarks and gluons as degrees of freedom and applying perturbation theory. At low-momentum scales the theory is strongly coupled so that hadronic degrees of freedom and nonperturbative methods must be used. It has therefore been very surprising that examples in which the behavior of low$Q^{2}$ cross sections can be related through suitable averaging procedures to those at high $Q^{2}$ actually exist. This phenomenon became known as quark-hadron duality. See the review [1].

\footnotetext{
*miller@phys.washington.edu
}

Published by the American Physical Society under the terms of the Creative Commons Attribution 4.0 International license. Further distribution of this work must maintain attribution to the author(s) and the published article's title, journal citation, and DOI. Funded by SCOAP ${ }^{3}$.
Here we focus on deep-inelastic scattering from hadrons. Bloom and Gilman [2,3] studied data from the early deep inelastic (DIS) structure experiments at SLAC. They found that the inclusive structure function measured at low values of the hadronic final state mass, $W$, generally follows a curve that describes data at large values of $W$. In particular, average values of the low $W$ cross sections were found to be approximately equal to that of the high $W$ cross section, which approximately obeys Bjorken scaling. Moreover, averages of low $Q^{2}$ data over specific kinematic regions were found to be equal to the high $W$ cross section.

Bloom-Gilman duality was later examined in terms of an operator product expansion of moments of structure functions $[4,5]$. This work found a systematic classification of terms responsible for duality, but did not describe how the physics of resonances transforms into the physics of scaling. The subject of Bloom-Gilman duality was largely ignored for about 20 years.

The availability of high-luminosity beams of electrons at Jefferson Laboratory allowed the subject to be studied in great detail. A striking finding was that Bloom-Gilman 
duality appears to work at values of $Q^{2}$ as low as about $1 \mathrm{GeV}^{2}$ or less [6-8].

Finding an elementary understanding of the origins of Bloom-Gilman duality has been elusive because it involves trying to build up structure function that is independent of $Q^{2}$ (except for the logarithmic corrections of QCD) entirely out of resonances, each of which is described by a form factor that falls rapidly with $Q^{2}$.

The description of Bjorken scaling in DIS structure functions is most simply formulated in terms of the quarkparton model, which is well understood in terms of a twist expansion, but the physical final state is composed of hadrons. The validity of both approaches indicates that describing DIS in terms of hadronic degrees of freedom should be possible. One of the central mysteries of stronginteraction physics is how scattering from confined bound states of quarks and gluons can be consistent with Bjorken scaling, a property associated with free quarks.

Previous approaches to understanding the origins of Bloom-Gilman duality, reviewed in [1], include QCD in $1+1$ dimensions [9], phenomenological approaches [10,11], harmonic oscillator models [12-15], and other models [16-18]. Nonrelativistic potential models can describe or represent confining systems with an infinite number of bound states, thereby indicating how it is that Bloom-Gilman scaling may arise, but are not properly relativistic. This means that none obtain Lorentz invariant quark distributions that have the correct support properties of being nonzero only in the region where Bjorken $x$ varies between 0 and 1 . Understanding Bloom-Gilman duality requires models that are both confining and relativistic.

We aim to understand Bloom-Gilman duality by using relativistic light-front wave functions obtained from lightfront holographic QCD, an approach defined in the review [19] that provides a relativistic treatment of confined systems. We briefly summarize following Ref. [19]. Light-front quantization is a relativistic, frame independent approach to describing the constituent structure of hadrons. The simple structure of the light-front (LF) vacuum allows an unambiguous definition of the partonic content of a hadron in QCD and of hadronic light-front wave functions, the underlying link between large distance hadronic states and the constituent degrees of freedom at short distances. The QCD light-front Hamiltonian $H_{\mathrm{LF}}$ is constructed from the QCD Lagrangian using the standard methods of quantum field theory [20]. The spectrum and light-front wave functions of relativistic bound states are obtained from the eigenvalue equation $H_{\mathrm{LF}}|\psi\rangle=M^{2}|\psi\rangle$. It becomes an infinite set of coupled integral equations for the LF components in a complete basis of noninteracting $n$-particle states, with an infinite number of components. This provides a quantum-mechanical probabilistic interpretation of the structure of hadronic states in terms of their constituents at the same light-front time $x^{+}=x^{0}+x^{3}$, the time marked by the front of a light wave [21]. The
Hamiltonian eigenvalue equation in the light front is frame independent. The matrix diagonalization [20] of the LF Hamiltonian eigenvalue equation in four-dimensional spacetime has not yet been achieved because of various technical difficulties and because of a lack of understanding of the fundamental mechanism of confinement. Therefore other methods and approximations are needed to truly understand the nature of relativistic bound states in the strong-coupling regime of QCD.

To a first semiclassical approximation, where quantum loops and quark masses are not included, the relativistic bound-state equation for light hadrons can be reduced to an effective LF Schrödinger equation. The technique is to identify the invariant mass of the constituents as a key dynamical variable. The invariant mass measures the offshellness in the LF kinetic energy, so that it is the natural variable to characterize the hadronic wave function. In conjugate position space, the relevant dynamical variable is an invariant impact kinematical variable $\zeta$, which measures the separation of the partons within the hadron at equal lightfront time [22]. Thus, the multiparton problem in QCD is reduced, in a first semiclassical approximation, to an effective one-dimensional quantum field theory by properly identifying the key dynamical variable. As a result, complexities of the strong interaction dynamics are hidden in an effective potential $U(\zeta)$, but the central question-how to derive the confining potential from QCD-remains open.

It is remarkable that in the semiclassical approximation described above, the light-front Hamiltonian has a structure which matches exactly the eigenvalue equations in anti-de Sitter (AdS) space [19]. This offers the possibility to explicitly connect the AdS wave function $\Phi(z)$ to the internal constituent structure of hadrons. In fact, one can obtain the AdS wave equations by starting from the semiclassical approximation to light-front QCD in physical spacetime. This connection yields a relation between the coordinate $z$ of AdS space with the impact LF variable $\zeta$ [22], thus giving the holographic variable $z$ a precise definition and intuitive meaning in light-front QCD.

Light-front holographic methods were originally introduced $[23,24]$ by matching the electromagnetic current matrix elements in AdS space [25] with the corresponding expression derived from light-front quantization in physical spacetime [26,27]. It was also shown that one obtains identical holographic mapping using the matrix elements of the energy-momentum tensor [28] by perturbing the AdS metric around its static solution [29], thus establishing a precise relation between wave functions in AdS space and the light-front wave functions describing the internal structure of hadrons.

The light-front wave functions that arise out of this lightfront holographic approach provide a new way to study old problems that require the use of relativistic-confining quark models. The study of Bloom-Gilman duality is an excellent example of such a problem. 
The present treatment is outlined next. Section II is concerned with general definitions related to DIS for spinless targets. Separate expressions for structure functions using both quark and hadronic degrees of freedom are presented, with the fundamental aim of the paper to relate the two. The global and local forms of Bloom-Gilman duality are reviewed briefly in Sec. III. Section IV is concerned with presenting the main features of light-front wave functions for two-parton systems that are obtained from the $z-\zeta$ connection. The valence quark number sum rule is discussed as a new global duality in Sec. V. This relation is satisfied in models that provide a complete set of wave functions. The existing light-front wave functions, summarized in Ref. [19], are found to be incomplete in Sec. VI. This is because excitations in the longitudinal degree of freedom are not incorporated. Completeness is implemented by including a longitudinal confining potential in Sec. VII. Evaluations of transition form factors are presented in Sec. VIII. The two-parton model is shown to violate both the global and the local forms of BloomGilman duality in Secs. IX and X. The model's quark distributions are evaluated in Sec. XI. The results are summarized and discussed in Sec. XII.

\section{DEEP INELASTIC SCATTERING FROM SPIN ZERO TARGET-GENERAL PRELIMINARIES}

The hadronic tensor for a spin-less or spin-averaged target is given by

$$
\begin{aligned}
W^{\mu \nu}= & \left(p^{\mu}-\frac{p \cdot q q^{\mu}}{q^{2}}\right)\left(p^{\nu}-\frac{p \cdot q q^{\nu}}{q^{2}}\right) \frac{W_{2}}{M^{2}} \\
& +\left(g^{\mu \nu}-\frac{q^{\mu} q^{\nu}}{q^{2}}\right) W_{1},
\end{aligned}
$$

with $p$ as the target four-momentum $\left(p^{2}=M^{2}\right)$ and $q$ that of the virtual photon. The quantity $W^{\mu \nu}$ is the matrix element of a current-current correlation function:

$$
W^{\mu \nu}=\frac{1}{4 \pi} \int d^{4} \xi e^{i q \cdot \xi}\left\langle p\left|j^{\mu}(\xi) j^{\nu}(0)\right| p\right\rangle,
$$

with normalization $\left\langle p^{\prime} \mid p\right\rangle=2 p^{+} \delta\left(p^{+}-p^{\prime+}\right)(2 \pi)^{3} \times$ $\delta\left(\mathbf{p}-\mathbf{p}^{\prime}\right)$. At large values of $Q^{2}$, the correlation is along the light cone. For spacelike four momenta $q^{\mu}$ one may choose the $z$ axis such that $q^{+}=0$, for spacelike values of $q^{\mu}$. This enables the use of the Drell-Yan formula [30] for form factors. With this notation $q^{3}=-\nu,|\mathbf{q}|=Q$, $\mathbf{q}^{2}=Q^{2}, \vec{q}^{2}=\nu^{2}+Q^{2}$. Here boldface indicates transverse vectors and $\vec{q}$ is the three-dimensional vector.

\section{A. Quark degrees of freedom}

The expression Eq. (2) can be handled by turning the product of currents into a commutator and then making the operator product expansion. The resulting leading-twist contribution to the quark distribution for a quark, $q(x)$ is given by

$q(x)=\int \frac{d x^{-}}{4 \pi} e^{i x P^{+} x^{-}}\left\langle p\left|\bar{\psi}\left(-x^{-} / 2\right) \gamma^{+} \psi\left(x^{-} / 2\right)\right| p\right\rangle$,

where $\psi$ is a quark-field operator, the notation $x^{-} / 2$ refers to $x^{\mu}=\left(0,-x^{-} / 2, \mathbf{0}\right)$, and $x=Q^{2} / 2 p \cdot q$. Scale dependence due to QCD evolution is omitted in this paper. Equation (3) is understood as involving wave functions evaluated at a given momentum scale. Thus the structure functions discussed reflect the intrinsic bound-state structure of the hadrons, and thus apply only at low resolution scales. Bloom and Gilman did not consider QCD evolution in their work. Accordingly such an effect is not a part of the present initial modern analysis.

One extracts $W_{2}$ from $W^{\mu \nu}$ by using

$$
W_{2}=W^{++} \text {. }
$$

At leading twist

$$
W_{2}=2 M^{2} \frac{x}{p \cdot q} W_{1},
$$

where $M$ is the ground state mass.

\section{B. Hadronic degrees of freedom}

An expression for $W^{++}$can be obtained using hadrons by inserting a complete set of hadronic states $|X\rangle$ between the current operators in Eq. (2). The use of $\left\langle p\left|j^{\mu}(\xi)\right| X\right\rangle=e^{i\left(p-p_{X}\right) \cdot \xi}\left\langle p\left|j^{\mu}(0)\right| X\right\rangle$, taking $\mu=+, \nu=+$ and integrating over $\xi^{+}$, yields

$W^{++}=\sum_{X} \frac{1}{2 E_{X}} \delta\left(p^{-}+q^{-}-p_{X}^{-}\right)\left|\left\langle X, x\left|j^{+}(0)\right| p\right\rangle\right|^{2}$,

in which it is understood that $\vec{p}_{X}=\vec{p}+\vec{q}=\vec{q}$ in the lab frame. The notation $X, x$ is meant to include all degenerate states of the same angular momentum. The factor of $2 E_{X}$ in the denominator comes from the relativistic normalization of states. The matrix elements of $j^{+}(0)$ are proportional to form factors,

$$
\left|F_{X, 0}\right|^{2} \equiv \sum_{x}\left|\left\langle X, x\left|\frac{j^{+}(0)}{2 p^{+}}\right| p\right\rangle\right|^{2},
$$

with $p_{X}^{+}=p^{+}+q^{+}=p^{+}$. The matrix element involves only internal coordinates of the wave functions, and 0 denotes the ground state in the lab frame with $\vec{p}=0$.

The argument of the delta function appearing in Eq. (6) can be expressed in more detail as 


$$
\begin{aligned}
p^{-}+q^{-}-p_{X}^{-} & =M+\nu-E_{X}(\vec{q}) \\
& =E_{0}+\nu-\sqrt{M_{X}^{2}+Q^{2}+\nu^{2}},
\end{aligned}
$$

where $M$ is the ground state energy. Then using $W^{++}=W_{2}$, we find

$$
\begin{aligned}
& W_{2}= 4 M^{2} \sum_{X} \frac{1}{2 \sqrt{M_{X}^{2}+Q^{2}+\nu^{2}}} \\
& \times \delta\left(M+\nu-\sqrt{M_{X}^{2}+Q^{2}+\nu^{2}}\right)\left|F_{X}\right|^{2} \\
&=4 M^{2} \sum_{X} \delta\left(M^{2}-M_{X}^{2}+Q^{2}\left(\frac{1}{x}-1\right)\right)\left|F_{X}\right|^{2} .
\end{aligned}
$$

The study of Bloom-Gilman duality involves relating the quark Eq. (3) and hadronic expressions Eq. (10).

\section{BLOOM-GILMAN DUALITY}

Bloom and Gilman found that the structure function in the resonance region, $W<2 \mathrm{GeV}$, was roughly equivalent on average to the scaling one, with averages obtained over the same region of the scaling variable:

$$
\omega^{\prime}=\frac{2 M \nu+M^{2}}{Q^{2}}=1+\frac{W^{2}}{Q^{2}}=\frac{1}{x}+\frac{M^{2}}{Q^{2}},
$$

where the invariant energy, $W$, is given by $W^{2}=$ $(p+q)^{2}=M^{2}+2 M \nu-Q^{2}$, which is the square of the mass of a resonance that is excited. Application of Eq. (11) requires that $\omega^{\prime}>0$ and $x>0$, so that $Q^{2}>M^{2}$. Bloom and Gilman noted that their sum rule is not valid if $Q^{2}$ is much less than $1 \mathrm{GeV}^{2}$.

Bloom and Gilman found that the data from the resonance region at low $Q^{2}$ oscillate around the scaling curve, with averages that are equal to the scaling curve. Furthermore the resonances move to lower values of $\omega^{\prime}$ (higher values of $x$ ) with increasing $Q^{2}$. These observations were repeated at Jefferson Lab.

Bloom and Gilman quantified their studies by observing the validity of a sum rule:

$\frac{2 M}{Q^{2}} \int_{0}^{\nu_{m}} d \nu \nu W_{2}\left(\nu, Q^{2}\right)=\int_{1}^{1+W_{m}^{2} / Q^{2}} d \omega^{\prime} \nu W_{2}\left(\omega^{\prime}\right)$,

where $W_{2}\left(\omega^{\prime}\right)$ is the scaling function obtained at large values of $Q^{2}$. The upper limit on the integration over $\nu$, $\nu_{m}=\left(W_{m}^{2}-M^{2}+Q^{2}\right) / 2 M$ corresponds to the maximum value of $\omega^{\prime}=1+W_{m}^{2} / Q^{2}$, where $W_{m} \approx 2 \mathrm{GeV}$. The validity of this equation is known as global duality.

Local duality is said to exist if the equality of the averaged resonance and scaling functions holds over restricted regions of $W$. BG obtained an explicit expression by taking the difference between two versions of Eq. (12) with different upper limits of integration,

$$
\frac{2 M}{Q^{2}} \int_{\nu_{a}}^{\nu_{b}} d \nu \nu W_{2}\left(\nu, Q^{2}\right)=\int_{1+W_{b}^{2} / Q^{2}}^{1+W_{a}^{2} / Q^{2}} d \omega^{\prime} \nu W_{2}\left(\omega^{\prime}\right) .
$$

\section{SOFT-WALL LIGHT-FRONT WAVE FUNCTIONS IN LIGHT-FRONT HOLOGRAPHIC QCD}

Consider a hadronic bound state of two constituents, each of vanishing mass. We follow the argument of [19]. The eigenmasses are given by

$$
\begin{aligned}
M^{2}= & \int_{0}^{1} \int \psi^{*}(x, \mathbf{k})\left[\frac{-\nabla_{\mathbf{k}}^{2}}{x(1-x)}\right] \psi(x, \mathbf{k}) d x \frac{d^{2} \mathbf{k}}{16 \pi^{3}} \\
& + \text { interactions, } \\
= & \int_{0}^{1} \int \psi^{*}(x, \mathbf{b})\left[\frac{-\nabla_{\mathbf{b}}^{2}}{x(1-x)}\right] \psi(x, \mathbf{b}) d x \frac{d^{2} \mathbf{b}}{4 \pi} \\
& + \text { interactions, }
\end{aligned}
$$

where the relative coordinates are the transverse separation b and the momentum fraction $x$. The invariant mass $M_{q \bar{q}}^{2}=\frac{\mathbf{k}^{2}}{x(1-x)}$. The canonically conjugate impact space variable is $\zeta^{2}=x(1-x) \mathbf{b}^{2}$. To a first approximation LF dynamics depend only on $M_{q \bar{q}}^{2}$ or $\zeta$, and the dynamical properties are encoded in the hadronic wave function $\phi(\zeta)$. Following standard procedure solutions in the product form,

$$
\psi(x, \zeta, \phi)=e^{i L_{z} \varphi} X(x) \frac{\phi(\zeta)}{\sqrt{2 \pi \zeta}},
$$

are sought. The quantity $L_{z}$ is the longitudinal component of the orbital angular momentum, an integer that can be positive or negative or 0 .

One proceeds by writing the Laplacian operator in Eq. (14) in the polar coordinates $(\zeta, \phi)$ :

$$
\nabla_{\zeta}^{2}=\frac{1}{\zeta} \frac{d}{d \zeta}\left(\zeta \frac{d}{d \zeta}\right)+\frac{1}{\zeta^{2}} \frac{\partial^{2}}{\partial \varphi^{2}}
$$

Then with the normalization

$$
\langle X \mid X\rangle=\int_{0}^{1} \frac{d x}{x(1-x)} X^{2}(x)=1
$$

one finds that Eq. (14) becomes

$$
\begin{aligned}
M^{2}= & \int d \zeta \phi^{*}(\zeta)\left(-\frac{d^{2}}{d \zeta^{2}}-\frac{1-4 L^{2}}{4 \zeta^{2}}\right) \phi(\zeta) \\
& +\int d \zeta \phi^{*}(\zeta) U(\zeta) \phi(\zeta)
\end{aligned}
$$


in which an effective potential $U(\zeta)$ has been introduced to enforce confinement at some infrared scale, and $L$ is the absolute value of $L_{z}$.

The resulting wave equation is

$$
\left(-\frac{d^{2}}{d \zeta^{2}}-\frac{1-4 L^{2}}{4 \zeta^{2}}+U(\zeta)\right) \phi(\zeta)=M^{2} \phi(\zeta)
$$

The soft-wall model [31]

$$
U(\zeta)=\kappa^{4} \zeta^{2}
$$

where $\kappa$ is the strength of the confinement, is used here. This form was used in [32]. Later work [33,34] introduced a constant term that depends on $L$, and relations between the baryon and meson spectrum were obtained. These results were derived from superconformal algebra. In line with our goal of examining Bloom-Gilman duality, the earlier form is used here to avoid a zero in the ground state mass.

The function $X(x)$ is to be determined. A popular choice has been to simply use $X(x)=\sqrt{x(1-x)}$. This is followed here. We'll show below that this choice does not yield a complete set of wave functions in threedimensional space.

The associated eigenvalues are

$$
M_{n L}^{2}=\kappa^{2}(4 n+2 L+2) .
$$

The light-front wave function [Eq. (15)] is obtained by solving Eq. (19) with the soft-wall potential of Eq. (20), and using $\zeta^{2}=x(1-x) b^{2}$ with the result

$$
\begin{aligned}
\psi_{n L_{z}}(\mathbf{b}, x)= & A_{n L} \sqrt{x(1-x)} \frac{e^{i L_{z} \varphi}}{\sqrt{2 \pi}} e^{-\kappa^{2} b^{2} x(1-x) / 2} \\
& \times(\sqrt{x(1-x)} b)^{L} L_{n}^{L}\left(\kappa^{2} b^{2} x(1-x)\right),
\end{aligned}
$$

where $b$ is the transverse radius, $\varphi$ is the transverse angle, and $x$ is the plus-momentum ratio of one of the quarks. The factor normalizing the wave function to unity is $A_{n L}=\sqrt{2} \sqrt{\frac{n !}{(n+L) !}}$. The spin dependence is taken to be a simple delta function, setting the helicity of the antiquark to the negative of the quark $[24,35]$. The action of the $\gamma^{+}$ operator in $j^{+}$conserves the spin of the struck quark so that all of the states, $X$ entering in Eq. (10), have the same spin wave function.

The next step is to evaluate the transition form factors. For a quark-antiquark system, of unit charge, with the given space and spin dependence, in the Drell-Yan frame, these are given by the expression

$$
\begin{aligned}
F_{X 0}(\mathbf{q}) \equiv & F_{n L_{z}}(\mathbf{q})=\int_{0}^{1} d x \int d^{2} \mathbf{b} e^{i \mathbf{q} \cdot \mathbf{b}(1-x)} \\
& \times \psi_{n L_{z}}^{*}(x, \mathbf{b}) \psi_{00}(x, \mathbf{b})
\end{aligned}
$$

with the notation $\left(\mathbf{q} \cdot \mathbf{q}=Q^{2}\right)$. This form factor is the same as would be obtained if only a single quark of unit charge interacts electromagnetically.

\section{VALENCE QUARK NUMBER SUM RULE-A NEW GLOBAL DUALITY}

In this two-parton model the quark and antiquark have the same relative wave function and therefore the same quark distribution. The form factor can be obtained as if only a single quark of unit charge interacts electromagnetically. In this case the form factors of Eq. (7) contribute to the single flavor $q\left(x, Q^{2}\right)$. This means that the sum over $X$ in Eq. (10) contributes only to $q(x)$, and we may take the quark distribution $q(x)$ as given by

$$
q(x)=W_{1}=\frac{p \cdot q}{2 M^{2} x} W_{2}=\frac{\nu}{2 M x} W_{2} .
$$

An interesting sum rule may be derived from the valence quark number sum rule:

$$
\int_{0}^{1} q(x) d x=1
$$

The integral over $x$ can be evaluated from the hadronic degrees of freedom, and using Eqs. (24) and (10) we examine the quark number sum rule to find

$q\left(x, Q^{2}\right)=\frac{Q^{2}}{x^{2}} \sum_{X} \delta\left(M^{2}-M_{X}^{2}+Q^{2}\left(\frac{1}{x}-1\right)\right)\left|F_{X}\right|^{2}$.

Noting that $F_{X}$ depends on $Q^{2}$, not on $x$, the integral over all values of $x$ yields

$$
\int_{0}^{1} q(x) d x=\sum_{X}\left|F_{X}\right|^{2} .
$$

Thus the quark number sum rule is satisfied if and only if the completeness relation

$$
\sum_{X}\left|F_{X}\right|^{2}=1
$$

is satisfied.

This result Eq. (27) amounts to a new form of global duality: At any given value of $Q^{2}$ the sum of the squares of all of the form factors, computed using hadron degrees of freedom, satisfies the quark number sum rule. The derivation presented here uses a single quark flavor and unit quark 
charge. The same global duality may be obtained in terms of the usual more general parton model conditions. It is a consequence of baryon number conservation.

\section{LACK OF COMPLETENESS}

In the current model the sum appearing in Eq. (28) is given by

$$
S(Q)=\sum_{n=0}^{\infty} \sum_{L_{z}=-\infty}^{\infty}\left|F_{n L_{z}}(Q)\right|^{2} .
$$

Satisfying the sum rule requires $S(Q)=1$.

Let us evaluate this quantity. To see this more explicitly consider

$$
\begin{gathered}
S(Q) \equiv \sum_{n=0}^{\infty} \sum_{L_{z}=-\infty}^{\infty}\left|F_{n L_{z}}(Q)\right|^{2} \\
=\int d x \int d^{2} b e^{i \mathbf{q} \cdot \mathbf{b}(1-x)} \psi_{n, L_{z}}^{*}(x, \mathbf{b}) \psi_{00}(x, \mathbf{b}) \\
\times \int d x^{\prime} \int d^{2} r^{\prime} e^{-i \mathbf{q} \cdot \mathbf{b}^{\prime}\left(1-x^{\prime}\right)} \psi_{n, L_{z}}\left(x^{\prime}, \mathbf{b}^{\prime}\right) \psi_{00}\left(x^{\prime}, \mathbf{b}^{\prime}\right) .
\end{gathered}
$$

Change variables to $\zeta=\mathbf{b} \sqrt{x(1-x)}$. Then use $d^{2} b x(1-$ $x)=d^{2} \zeta$ and define

$$
\phi_{n L_{z}}(\zeta) \equiv \frac{\psi_{n L_{z}}(x, \zeta)}{\sqrt{x(1-x)}}
$$

The $\phi_{n L_{z}}(\zeta)$ are standard two-dimensional (2D) harmonic oscillator wave functions. Then

$$
\begin{aligned}
S(Q)= & \sum_{n=0}^{\infty} \sum_{L_{z}=-\infty}^{\infty} \int d x \int d^{2} \zeta e^{i \mathbf{q} \cdot \zeta \sqrt{\frac{1-x}{x}}} \phi_{n, L_{z}}^{*}(\zeta) \phi_{00}(x, \zeta) \\
& \times \int d x^{\prime} \int d^{2} b^{\prime} e^{-i \mathbf{q} \cdot \zeta^{\prime} \sqrt{\frac{1-x^{\prime}}{x^{\prime}}}} \phi_{n, L_{z}}\left(\zeta^{\prime}\right) \phi_{00}\left(\zeta^{\prime}\right) .
\end{aligned}
$$

But standard 2D harmonic oscillator wave functions obey

$$
\sum_{n=0}^{\infty} \sum_{L_{z}=-\infty}^{\infty} \phi_{n, L_{z}}^{*}(\zeta) \phi_{n, L_{z}}\left(\zeta^{\prime}\right)=\delta\left(\zeta-\zeta^{\prime}\right),
$$

so that

$$
S(Q)=\int d x \int d x^{\prime} \int d^{2} \zeta e^{i \mathbf{q} \cdot \zeta\left(\sqrt{\frac{1-x}{x}}-\sqrt{\frac{1-x^{\prime}}{x^{\prime}}}\right)}\left|\phi_{00}(\zeta)\right|^{2} .
$$

The integral over $\zeta$ can be done so that

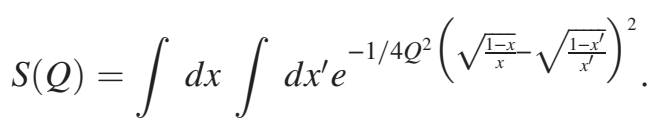

The validity of the sum rule requires that $S(Q)=1$ for all values of $Q$. This is true only for $Q=0$. For all other values, it is manifest that $S(Q>0)<1$. Completeness is not satisfied. Numerical work shows that $\lim _{Q \rightarrow \infty} S(Q) \sim \frac{1}{Q}$, and one can analytically show $\lim _{Q \rightarrow \infty} S(Q) \approx \frac{\pi^{3 / 2}}{8 Q}$ by using the method of steepest descent. The net result is that instead of unity one gets 0 for large enough values of $Q$.

This study of $S(Q)$ shows that Eq. (29) is NOT satisfied in the current model. This is because the wave functions given above in Eq. (22) are complete only in the twodimensional $\zeta$ space, not in the $x$ space.

\section{IMPLEMENTING COMPLETENESS}

The functions of Eq. (22) do not form a complete set over the three-dimensional space $x, \mathbf{r}$ because $X(x)=$ $\sqrt{x(1-x)}$ is not a complete set of wave functions in $x$ space. The need to include excitations of longitudinal modes was noted in Ref. [36].

Here we construct a set of wave functions in $x$ space in which the given $X(x)$ corresponds to a ground state. This is done by generalizing the interaction to include a longitudinal potential

$$
U_{\mathcal{L}}(x)=-\lambda \kappa^{2} \frac{d}{d x} x(1-x) \frac{d}{d x} .
$$

This interaction is approximately harmonic oscillator potential in the longitudinal variable $\tilde{z}$ of [37], with $\tilde{z}^{2}=-\frac{\partial^{2}}{\partial x^{2}}$. The factor $x(1-x)$ appears here and in the transverse soft-wall potential of Eq. (20) when it is expressed in terms of light-front variables.

This added potential gives a contribution to the square of the mass, $M_{\mathcal{L}}^{2}$ given by

$M_{\mathcal{L}}^{2}=-\lambda \kappa^{2} \int_{0}^{1} d x \frac{X(x)}{\sqrt{x(1-x)}} \frac{d}{d x} x(1-x) \frac{d}{d x} \frac{X(x)}{\sqrt{x(1-x)}}$,

where $\lambda$ is a dimensionless number. The normalized solutions to the related differential equation are the functions

$$
\frac{X(x)}{\sqrt{x(1-x)}}=\sqrt{2 \mathcal{L}+1} P_{\mathcal{L}}(2 x-1),
$$

where $P_{\mathcal{L}}$ is a Legendre polynomial (with $\mathcal{L}$ an integer) with eigenvalues $M_{\mathcal{L}}^{2}=\lambda \kappa^{2} \mathcal{L}(\mathcal{L}+1)$. For 
$\mathcal{L}=0, X(x)=\sqrt{x(1-x)}$ so previous results for the spectrum are preserved and correspond to modes with $\mathcal{L}=0$.

The value of $\lambda$ should be determined by an appropriate symmetry. We hope that the need to satisfy completeness will lead to future work on this topic and leave finding such a symmetry for future work and future workers. Our only purpose here is to study Bloom-Gilman duality. A complete set of states is needed to do that, as shown in Sec. V. Here we note that setting $\lambda=2$ means the lowenergy part of the spectrum obtained in Refs. $[33,34]$ is not changed.

The net result is that light-front wave functions that provide the necessary complete set are given by

$$
\Psi_{n L_{z} \mathcal{L}}(x, \varphi, \mathbf{r})=\psi_{n L_{z}}(\mathbf{r}, x) \sqrt{x(1-x)} P_{\mathcal{L}}(2 x-1) .
$$

Using $\Psi_{n, L_{z} \mathcal{L}}$ satisfies the completeness relation in the $(x, \mathbf{b})$ space because the Legendre polynomials form a complete set of orthogonal polynomials.

The spectrum is now given by

$$
M_{n L \mathcal{L}}^{2}(\lambda)=\kappa^{2}(4 n+2 L+2 \mathcal{L}(\mathcal{L}+1)+2) .
$$

\section{TRANSITION FORM FACTORS}

The transition form factors must be evaluated in preparation for calculating $W_{2}$ as given by the hadronic expression of Eq. (10). Specific expressions for these form factors have not been presented previously.

Let us start with the $\mathcal{L}=0$ sector. The angular integral appearing in $d^{2} b$ can be done in closed form with the result

$$
F_{n L_{z}}(\mathbf{q})=(-i)^{L_{z}} 2 \sqrt{\frac{n !}{(n+L) !}} \int_{0}^{1} d x \int_{0}^{\infty} d z z^{L+1} e^{-z^{2}} L_{n}^{L}\left(z^{2}\right) J_{L}\left(Q z \sqrt{\frac{1-x}{x}}\right) .
$$

Evaluation leads to

$$
F_{n L_{z}}(Q)=(-i)^{L_{z}} \sqrt{\frac{1}{n !(n+L) !}} \int_{0}^{1} d x\left(\frac{Q}{2 \kappa} \sqrt{\frac{1-x}{x}}\right)^{N} e^{-\frac{Q^{2} 1-x}{4 \kappa^{2} x}}
$$

in which $|\mathbf{q}|=Q$ is used and $N \equiv 2 n+L$.

This integral is found analytically in terms of an incomplete Gamma function as

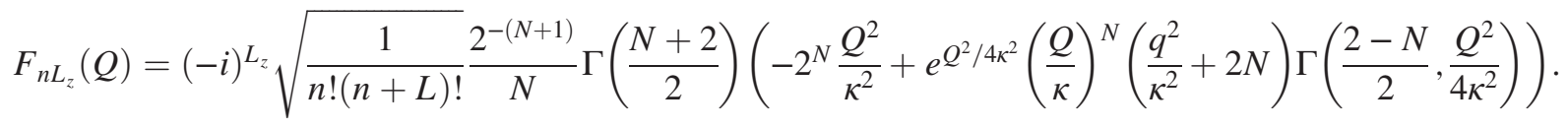

An alternate evaluation of the integral that allows the asymptotic limit of the transition form factors to be obtained is given next.

Define

$$
I_{N} \equiv \int_{0}^{1} d x\left(\frac{Q}{2 \kappa} \sqrt{\frac{1-x}{x}}\right)^{N} e^{-\frac{Q^{2} 1-x}{4 \kappa^{2} x}}
$$

with

$$
F_{n L_{z}}(Q)=(-i)^{L_{z}} \sqrt{\frac{1}{n !(n+L) !}} I_{N}(Q)
$$

Let $K \equiv N / 2, z \equiv \frac{Q^{2}}{4 \kappa^{2}}$, and $u \equiv \frac{1-x}{x}$. Then

$$
\begin{aligned}
I_{K}(z) & =z^{K} \int_{0}^{\infty} \frac{d u}{(1+u)^{2}} u^{K} e^{-z u} \\
& =z \Gamma(K)\left(e^{z}(K+z) E_{K}(z)-1\right), \quad K>0,
\end{aligned}
$$

with

$$
E_{K}(z)=\int_{1}^{\infty} \frac{e^{-z t}}{t^{K}} d t
$$

and

$$
I_{0}(z)=\int_{0}^{\infty} \frac{d u}{(1+u)^{2}} e^{-z u}=1-z e^{z} \int_{z}^{\infty} \frac{e^{-t}}{t} d t .
$$

It is useful to consider the asymptotic values of the transition form factors for fixed values of $K$ and $z \gg K$. This is because Bloom and Gilman found that one of the requirements for local duality is that all of the transition form factors have the same dependence on $Q^{2}$. First, note that for integer values of $K$ one may write

$$
I_{K}(z)=z^{K}\left(-\frac{\partial}{\partial z}\right)^{K} I_{0}(z) .
$$


The asymptotic limit of $z \rightarrow \infty$ is obtained by integration by parts of the first of the expressions for $I_{0}(z)$ :

$I_{0}(z)=\frac{-1}{z} \int_{0}^{\infty} \frac{d u}{(1+u)^{2}} \frac{d}{d u} e^{-z u}=\frac{1}{z}+\mathcal{O}\left(\frac{1}{z^{2}}+\cdots\right)$.

Keeping the leading term and carrying out the derivatives of Eq. (50) gives

$$
I_{K}(z) \sim \frac{K !}{z}=\frac{K \Gamma(K)}{z},
$$

which leads to the same result as taking the limit of Eq. (44). The latter expression works for half-integer values of $L$.

The net result is that as $Q^{2}$ approaches $\infty$ for $z=Q^{2} / 4 \kappa^{2} \gg K$

$$
\lim _{Q^{2} \rightarrow \infty} F_{n L_{z}}\left(Q^{2}\right)=\frac{4\left(n+\frac{L}{2}\right) !}{Q^{2}} i^{L_{z}} \frac{1}{\sqrt{n !(n+L) !}} .
$$

This limit is not to be used in evaluating the form factors necessary to compute $q(x)$ because the assumption that $z \gg K$ is violated in doing the sum over states which goes in principle to infinite values of $K$.

The universal $1 / Q^{2}$ behavior shown in Eq. (53) occurs because the integral of Eq. (49) is dominated by small values of $u$ which corresponds to large values of $x$. This model presents an example of the Feynman model [38] of form factors in which the dominant contributions to the form factors occur when one quark, carrying nearly all of the momentum of the hadron, is turned around by the virtual photon.

\section{A. $\mathcal{L}>\mathbf{0}$}

We now need

$$
I_{K, \mathcal{L}}(z) \equiv z^{L} \int_{0}^{\infty} \frac{d u}{(1+u)^{2}} u^{K} e^{-z u} P_{\mathcal{L}}\left(\frac{1-u}{1+u}\right)
$$

Using the same arguments as before, we can show that

$$
\lim _{z \rightarrow \infty} I_{K, \mathcal{L}}(z)=\frac{K \Gamma(K)}{z} .
$$

This is because the Legendre polynomial in Eq. (54) is unity at $u=0$.

We have not been able to obtain a closed form expression for a general value of $\mathcal{L}$. Instead, we evaluate term by term

$$
\begin{aligned}
I_{K, 1}(z)= & -z \Gamma(K)\left(e^{z}\left((K+z)^{2}+z\right) E_{K}(z)\right. \\
& -K-z-1), K>0),
\end{aligned}
$$

$$
\begin{aligned}
& I_{0,1}(z)=z\left(e^{z}(z+1) \int_{z}^{\infty} d t \frac{e^{-t}}{t}-1\right), \quad(57) \\
I_{K, 2}(z)= & z \Gamma(K)\left(e^{z}\left((K+z)\left((K+z)^{2}+3 z\right)+z\right) E_{K}(z)\right. \\
& \left.-K^{2}-K(2 z+1)-z(z+3)-1\right), \quad K>0,
\end{aligned}
$$

$I_{0,2}(z)=z\left(2+z-e^{z}(1+z(3+z)) \int_{z}^{\infty} d t \frac{e^{-t}}{t}\right)$.

Similar expressions can be obtained for any value of $\mathcal{L}$. The net result is that

$F_{n L_{z}, \mathcal{L}}(Q)=(-i)^{L_{z}} \sqrt{\frac{1}{n !(n+L) !}} \sqrt{2 \mathcal{L}+1} I_{K, \mathcal{L}}(Q)$,

with $K=n+L / 2$.

\section{BLOOM-GILMAN GLOBAL DUALITY IS NOT SATISFIED BY THIS MODEL}

Prior to evaluating $q(x)$ it is useful and possible to see if the sum rule of Eq. (12) is satisfied. The right-hand side of that equation is to be obtained by the high $Q^{2}$ expression for $q(x)$. This quantity is obtained from Eq. (3) by using the field expansion, in the standard expressions of the twoparton Fock-space component wave function. The result using the model of Sec. IV is that

$$
q(x)=\int d^{2} b\left|\psi_{00}(b, x)\right|^{2}=1 .
$$

This expression involves an integral over all values of $\mathbf{b}$ and therefore is equivalent to an integral over all values of $\mathbf{k}$. This latter integral corresponds to an infinite momentum transfer scale [35]. Thus QCD evolution of the distributions is not discussed further. The $Q^{2}$ dependence of $q\left(x, Q^{2}\right)$ of Eq. (26) arises from the $Q^{2}$ dependence of the resonance form factors.

The result $q(x)=1$ requires further comment because it displays the unrealistic nature of the present model. According to quark counting rules (see, e.g., [39]), the structure function of a two-parton state should fall as $1-x$ for large values of $x$, with QCD-DGLAP evolution providing a faster falloff. Bloom and Gilman [2,3] use quark counting rules in the form of the Drell-Yan-West [26,27] relations to understand their duality. As shown by Drell and Yan [26], a quark structure function varies at large $x$ as $(1-x)^{2 n-1}$ if the corresponding form factor $F\left(Q^{2}\right) \sim$ $\left(1 / Q^{2}\right)^{n}$ at large values of $Q^{2}$. The present model violates this relation because, as shown above, all of the form factors have the same asymptotic $1 / Q^{2}$ behavior, but the structure function is constant. In the present model, the 
asymptotic nature of the form factor is determined by the Feynman mechanism. The quark counting rules are based on vector meson exchange between the quarks, so that the important region occurs when all of the partons are on top of each other. Feynman [38] argued that is not realistic.

The validity of the Feynman vs quark-counting picture is one of the important issues related to color transparency [40], the interesting quantum invisibility predicted to occur as the result of vanishing of initial- or final-state interactions. Color transparency cannot occur if the Feynman mechanism is dominant [41].

The purpose of the present paper is to investigate the stated model. Although simple, it has the correct completeness and support properties that enable study of Bloom-Gilman duality. Therefore we investigate the sum rule Eq. (12) by using $q(x)=1$. This allows the right-hand side $\equiv R\left(Q^{2}\right)$ of Eq. (12) to be evaluated immediately:

$R\left(Q^{2}\right) \equiv \int_{1}^{1+W_{m}^{2} / Q^{2}} d \omega^{\prime} \nu W_{2}\left(\omega^{\prime}\right)=2 M \int_{1}^{1+W_{m}^{2} / Q^{2}} d \omega^{\prime} x$,

with $x=1 /\left(\omega^{\prime}-M^{2} / Q^{2}\right)$. Evaluation yields

$$
R\left(Q^{2}\right)=2 M \ln \frac{Q^{2}+W_{m}^{2}-M^{2}}{Q^{2}-M^{2}}
$$

and

$$
\lim _{Q^{2} \rightarrow \infty} R\left(Q^{2}\right)=2 M \frac{W_{m}^{2}}{Q^{2}} .
$$

This result of using the stated model is already in violation of the Bloom-Gilman condition that it be independent of $Q^{2}$. The independence could be obtained by taking $Q^{2}$ to infinity, so that $R \rightarrow 0$, and the violation of global duality is assured.

Next examine the left-hand side of Eq. (12) $\left[\equiv L\left(Q^{2}\right)\right]$. First, the lower limit on the integral over $\nu$ is determined by the largest value of $x(=1)$ for which $\nu W_{2}$ is nonzero. This is given by $Q^{2} /(2 M)$. Converting the integral over $\nu$ to one over $x$ gives

$$
L\left(Q^{2}\right)=2 M \int_{\frac{Q^{2}}{\left(Q^{2}+W_{m}^{2}-M^{2}\right)}}^{1} \frac{d x}{x} q\left(x, Q^{2}\right)
$$

in which $q\left(x, Q^{2}\right)$ is to be obtained from the hadronic expression, Eq. (10). Simplifying the argument of the delta function leads to

$$
q\left(x, Q^{2}\right)=\sum_{X}\left|F_{X}(Q)\right|^{2} \delta\left(x-x_{X}\right),
$$

where $X \equiv\left(n, L_{z}, \mathcal{L}\right)$ and $x_{X}=\frac{Q^{2}}{Q^{2}+2 \kappa^{2}(2 n+L+\mathcal{L}(\mathcal{L}+1))}$.

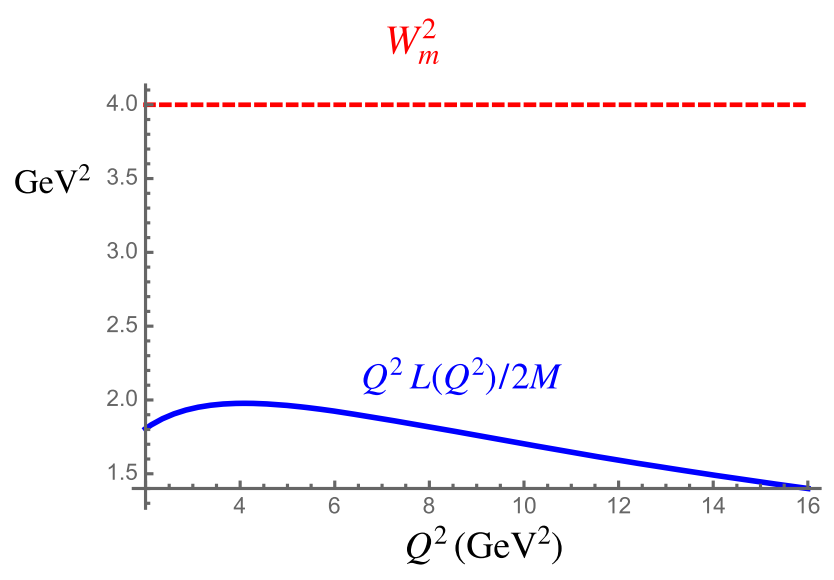

FIG. 1. $Q^{2} L\left(Q^{2}\right) / 2 M$ vs $W_{m}^{2}$.

Thus

$$
\begin{aligned}
L\left(Q^{2}\right)= & 2 M \sum_{n, L_{z}, \mathcal{L}} \frac{Q^{2}+2 \kappa^{2}(2 n+L+\mathcal{L}(\mathcal{L}+1))}{Q^{2}} \\
& \times F_{n L_{z} \mathcal{L}}^{2}\left(Q^{2}\right) \Theta\left(W_{m}^{2}-2 \kappa^{2}(2 n+L+\mathcal{L}(\mathcal{L}+1)) .\right.
\end{aligned}
$$

If $R$ were equal to $L$, the sum appearing in Eq. (67) would need to vary as $1 / Q^{2}$. This seems unlikely because for finite values of $M_{X}^{2}$, each form factor varies as $1 / Q^{2}$, leading to an overall dependence varying approximately as $1 / Q^{4}$. The asymptotic limit is not precisely accurate, but nevertheless $L\left(Q^{2}\right)$ falls much faster than $1 / Q^{2}$.

This dependence is shown in Fig. 1. To obtain this figure we take $M^{2}=2 \kappa^{2}$, with $M$ the nucleon mass and $W_{m}=2 \mathrm{GeV}$, so that $2 n+L+2 \mathcal{L}(\mathcal{L}+1) \leq 4$. The result is shown in Fig. 1. Global duality would hold if $Q^{2} R\left(Q^{2}\right) / 2 M=Q^{2} L\left(Q^{2}\right) / 2 M$, i.e., if the blue and red curves were equal.

\section{BLOOM-GILMAN LOCAL DUALITY IS NOT SATISFIED BY THE MODEL}

Local duality is studied through Eq. (13). If the upper and lower limits are taken to encompass one resonance, $X$, this equation leads to the relation

$$
\frac{1}{x_{X}} F_{X}^{2}=\ln \frac{W_{+}^{2}+Q^{2}-M^{2}}{W_{-}^{2}+Q^{2}-M^{2}},
$$

with $W_{ \pm}=M_{X} \pm \gamma / 2$. Here the value of $\gamma$ can be any energy less than the minimum spacing between levels, $2 \kappa^{2}$. A first glance indicates that the relation Eq. (68) is not generally satisfied because the right-hand side depends on $\gamma$, but the left-hand side does not. Moreover, the right-hand side falls as $1 / Q^{2}$ for large values of $Q^{2}$, but the left-hand side falls as $1 / Q^{4}$. 


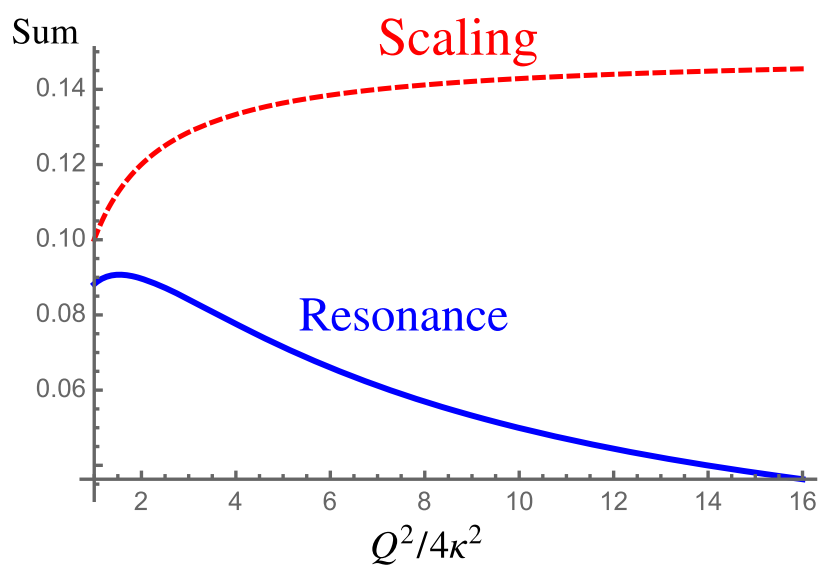

FIG. 2. Left- (blue, dashed, resonance curve) and right- (red, solid, scaling curve) hand sides of Eq. (68) times $Q^{2} / 4 \kappa^{2}$, with $\gamma=0.125 \kappa$.

An explicit calculation is made by choosing the lowest energy resonance with $n=0, L=1 . \mathcal{L}=0$ is an example. The results, shown in Fig. 2, are that indeed the local duality relation is not satisfied.

\section{EVALUATE $q\left(x, Q^{2}\right)$}

The quantity to evaluate is $q\left(x, Q^{2}\right)$ of Eq. (26). The need to include a nonzero width of the excited states in studying duality has been noted in $[12,13]$. Moreover, excited states do have nonzero widths. This is addressed next using a Breit-Wigner form.

The starting point is the function $\delta\left(M^{2}-M_{X}^{2}+\right.$ $Q^{2}(1 / x-1)=\delta\left(M_{X}^{2}-W^{2}\right)$, where $W^{2}=(p+q)^{2}$. The value of $x$ that yields a vanishing argument is defined to be $x_{X}$ with

$$
x_{X}\left(Q^{2}\right)=\frac{Q^{2}}{M_{X}^{2}-M^{2}+Q^{2}},
$$

showing that the contribution of a given resonance moves to larger values of $x$ as the value of $Q^{2}$ increases.

It is useful to relate $\delta\left(W^{2}-M_{X}^{2}\right)$ to a delta function $\delta\left(x-x_{X}\right)$, allowing a direct study of the new global duality of Sec. V. This may be done by first using $\delta\left(M_{X}^{2}-W^{2}\right)=\frac{1}{2 W} \delta\left(M_{X}-W\right)=\frac{1}{2 W} \lim _{\Gamma \rightarrow 0} \frac{1}{\pi} \frac{\Gamma / 2}{W-M_{X}+i \Gamma / 2}$.

Then apply a small-width approximation, $W-M_{X}=$ $\sqrt{Q^{2}\left(\frac{1}{x}-1\right)+M^{2}}-\sqrt{Q^{2}\left(\frac{1}{x_{X}}-1\right)+M^{2}} \approx-\left(x-x_{X}\right) \frac{Q^{2}}{2 M_{X} x_{X}^{2}}$ so that

$$
\begin{aligned}
\delta\left(M_{X}^{2}-W^{2}\right) & \rightarrow \frac{\Gamma_{X}}{\pi} \frac{M_{X} x_{X}^{4}}{\left.Q^{4}\left(x-x_{X}\right)^{2}+\Gamma_{X}^{2} x_{X}^{4} M_{X}^{2}\right)} \\
& \equiv \hat{\delta}\left(W^{2}-M_{X}^{2}\right) .
\end{aligned}
$$

The final step is to make the appearance of $\delta\left(x-x_{X}\right)$ explicit,
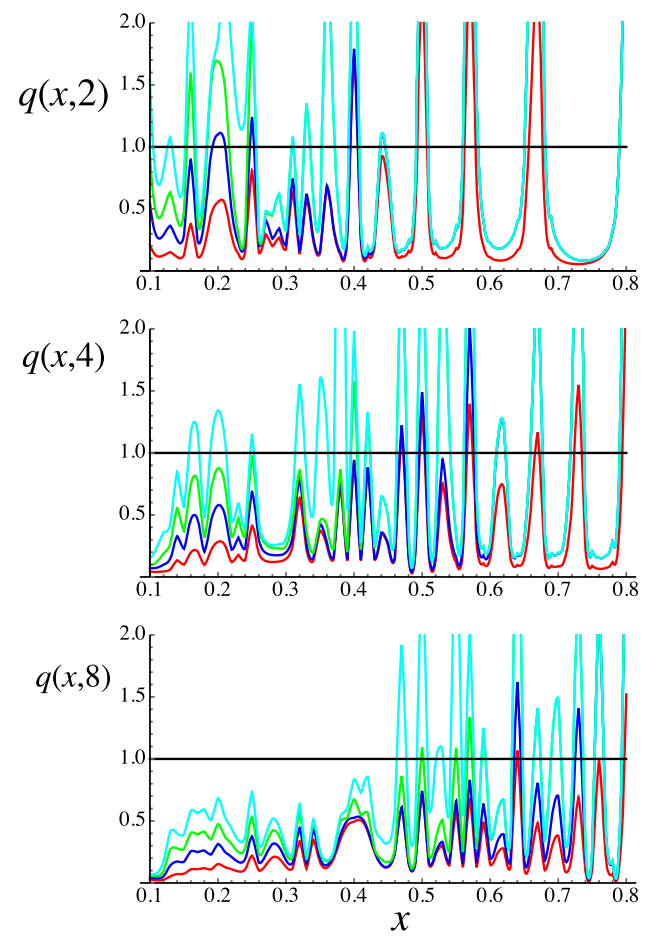

FIG. 3. $q(x, z)$ for $z=Q^{2} / 4 \kappa^{2}=2,4,8$. The four curves in each figure are $q_{\mathcal{L}=0}(x), q_{\mathcal{L}=0}(x)+q_{\mathcal{L}=1}(x), q_{\mathcal{L}=0}(x)+q_{\mathcal{L}=1}(x)+$ $q_{\mathcal{L}=2}(x), q_{\mathcal{L}=0}(x)+q_{\mathcal{L}=1}(x)+q_{\mathcal{L}=2}(x)+q_{\mathcal{L}=3}(x)$.

$\hat{\delta}\left(W^{2}-M_{X}^{2}\right)=\frac{1}{\pi} \frac{\varepsilon_{X}}{\left(x-x_{X}\right)^{2}+\varepsilon_{X}^{2}} \frac{x_{X}^{2}}{Q^{2}}=\hat{\delta}\left(x-x_{X}\right) \frac{x_{X}^{2}}{Q^{2}}$

with $\varepsilon_{X} \equiv \frac{\Gamma_{X} M_{X} x_{X}^{2}}{Q^{2}}$. Then

$q\left(x, Q^{2}\right)=\sum_{X} \frac{x_{X}^{2}}{x^{2}}\left|F_{X}\left(Q^{2}\right)\right| f \hat{\delta}\left(x-x_{X}\right) \equiv \sum_{\mathcal{L}=0}^{3} q_{\mathcal{L}}\left(x, Q^{2}\right)$.

The factor $f$ ensures that $\int_{0}^{1} d x \hat{\delta}\left(x-x_{X}\right)=1$, and $f$ approaches unity as $\varepsilon_{X}$ approaches 0 . The second form shows the sum of states with different values of $\mathcal{L}$. Each $q_{\mathcal{L}}\left(x, Q^{2}\right)$ includes a sum over values of $n$ and $L_{z}$. A maximum value of $\mathcal{L}=3$ is used. Although not complete, this is sufficient to display the main points and is necessary because an infinite number of values is needed for completeness.

The next step is to evaluate Eq. (72). Note that the sum over $X$ involves summing over $n, L_{z}= \pm L$ and $\mathcal{L}$, including all states The results of evaluating Eq. (72) are shown for $z=Q^{2} /(4 \kappa)=2,4,8$ in Fig. 3. This corresponds to $Q^{2}=8,16,32 \kappa^{2}=4,8,16 \mathrm{M}^{2}$. The width is taken as $\Gamma / \kappa=0.05=0.2 M / \sqrt{2}$. This is a constant width of space $66 \mathrm{MeV}$, with $M$ as the nucleon mass. In the spirit of the 

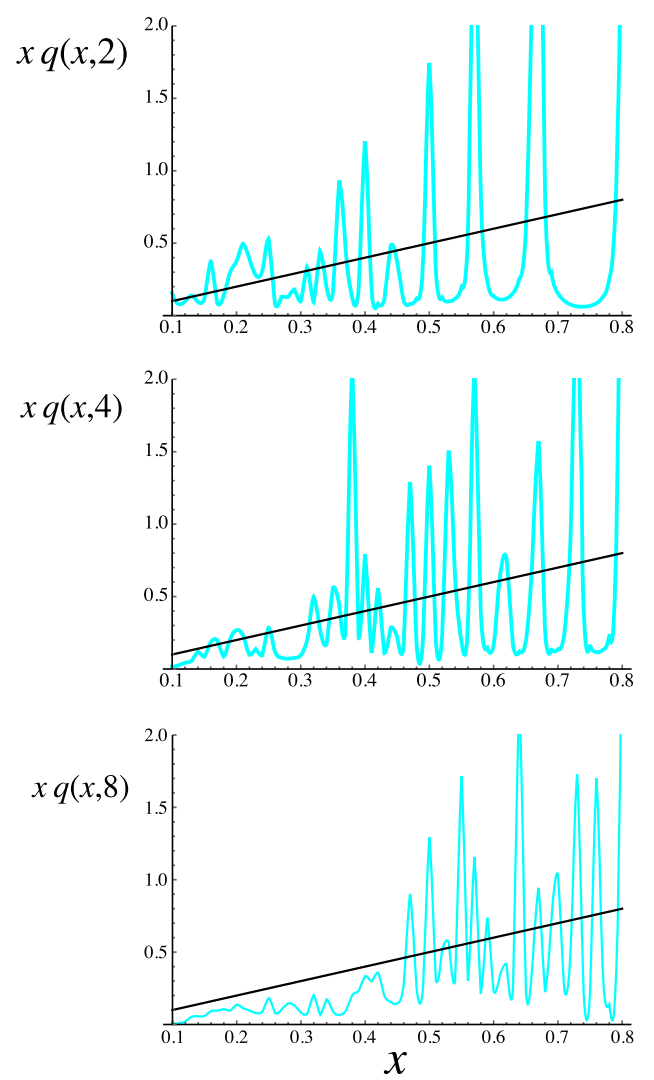

FIG. $\quad 4 . \quad x\left(q_{\mathcal{L}=0}(x, z)+q_{\mathcal{L}=1}(x)+q_{\mathcal{L}=2}(x)+q_{\mathcal{L}=3}(x)\right)$ for $z=Q^{2} / 4 \kappa^{2}=2,4,8$. The lines are drawn to guide the eye.

model, the width is taken to be small $[12,13]$. The maximum number of states in the sum Eq. (72) is increased until convergence is reached. In each figure the lowest curve is obtained using $\mathcal{L}=0$, and then the effects of $\mathcal{L}=1$ are added leading to the next lowest curve. Then the effects of adding states with $\mathcal{L}=2$ and $\mathcal{L}=3$ are included and result in two more curves.

Focusing first on $z=2$ and examining the lowest curve, one sees a spiky structure due to the resonance curves. Decreasing the value of the width leads to similar results, with narrower widths and higher peaks. The same pattern is obtained when adding the effects of states with $\mathcal{L}>0$, with different values of $M_{X}$, Eq. (41), leading to different peaks that increase the distribution function at low values of $x$, as expected from Eq. (69). The contributions of states with $\mathcal{L}>0$ do not contribute at higher values of $x$ due to the delta function appearing in Eq. (72). The solid line in each panel shows the scaling result $q(x)=1$. The resonance curves oscillate about the scaling curve for the larger values of $x$, but not for the lower ones.

The patterns for different values of $\mathcal{L}$ are also seen as the value of $z$ increases. A detailed difference is the effect at high values of $x$ is larger. Another is that the curves fall further below the scaling result, and the general tendency of the magnitude of $q\left(x, Q^{2}\right)$ to decrease as $Q^{2}$ increases is seen. This is another example of the model's failure to achieve local duality.

The Bloom and Gilman work found duality in $\nu W_{2}$ which is proportional to $x q(x)$. Plots of that quantity are shown in Fig. 4. The lines show $x q(x)=x$. This figure shows that $x q\left(x, Q^{2}\right)$ does approximately oscillate about a line. This is true, in part, because including the factor $x$ suppresses the region for which the result of using the sum of resonances, and Eq. (72) falls below $q(x)=1$. We denote this approximate oscillation as accidental duality, because the detailed evaluations of both global and local duality expressions (12) and (13) show a failure to achieve the necessary equality.

These presented results do not demonstrate that the use of hadronic degrees of freedom leads to the equality of the sum, Eq. (72) with the correct model quark distribution $q(x)=1$. This is expected because it is only possible to include a finite number of excitations $X$ and because of the necessity of including a nonzero width to obtain finite values of $q\left(x, Q^{2}\right)$. However, but they do show that adding intermediate states of higher and higher masses tends toward that direction. This is because adding states of higher mass tends to fill in gaps left by including only lower mass states.

\section{SUMMARY AND DISCUSSION}

Light-front wave functions motivated by holographic constructions are used to study Bloom-Gilman duality in this paper. Expressions for the structure functions in terms of quark Eq. (3) and hadronic Eq. (10) degrees of freedom (involving transition form factors) are presented, with an ultimate goal of obtaining a relationship between the two expressions. The specific two-parton model is defined in Sec. IV, with masses, Eq. (21), and light-front wave functions, Eq. (22), that had been obtained in the original work. Transition form factors are expressed, using the Drell-Yan frame, in terms of these wave functions in Eq. (23).

The valence quark-number sum rule is presented as a new form of global duality (integral over all values of $x$ between 0 and 1) in Sec. V, specifically in Eqs. (27) and (28). Using a complete set of hadronic states is necessary for this new global duality to be achieved, Eq. (28). That the original work does not provide a complete set is shown in Eq. (36). The lack of completeness arises from the choice in the original work to examine the presumably dominant configuration of the modes of lowest energy in which the longitudinal wave function of Eq. (15) is given by $X(x)=\sqrt{x(1-x)}$. This single form is not sufficient to provide a complete set in $x$-space. The lack of completeness is remedied by including a longitudinal potential, $U_{\mathcal{L}}(x)$, Eq. (37), where $\mathcal{L}$ is an integer quantum number, for which the stated $X(x)$ is the lowest energy mode with $\mathcal{L}=0$. The overall strength, $\lambda$, of $U_{\mathcal{L}}$ is arbitrarily chosen here. 
In principle, this parameter, along with the longitudinal potential, should be chosen by some symmetry principle that is unknown to us. The final result should achieve rotational invariance in the model, in the sense of having states with the proper degeneracy. This achievement is left as a goal of future research. Nevertheless, the present complete set is sufficient to carry out the specific purpose of this manuscript which is to examine Bloom-Gilman duality.

Given the complete set of wave functions, specific expressions for the transition form factors are obtained in Eq. (44), Eq. (46), and Eq. (60). All of the form factors fall as $1 / Q^{2}$, Eq. (52), at asymptotically large values. This behavior originates in the dominance of the Feynman mechanism for this model. That the dominant transition form factors have the same asymptotic dependence is one of the requirements to achieve Bloom-Gilman duality. However, the Drell-Yan connection between form factors and structure functions is also needed, and this feature is absent in the current model.

The model transition form factors are used to assess the validity of the global, Eq. (11), and local, Eq. (12), duality sum rules. The simplicity of the scaling quark distribution in the given model, $q(x)=1$, readily enables studies of these sum rules, with the result that both are not satisfied within the given model. See Figs. 1 and 2.

Evaluations of the hadronic expression for $q\left(x, Q^{2}\right)$, Eq. (72), are presented in the previous section; see Figs. 3 and 4 . The need to obtain noninfinite values to make plots of finite size mandates that the resonant states have at least a small width, as implemented in Eq. (71). The value of the width is chosen to be a small number $66 \mathrm{MeV}$. Detailed results depend upon the precise value, but the qualitative conclusions do not. The figures show that including the states with $\mathcal{L}>0$ are needed to approach Bloom-Gilman duality, but that this duality is not obtainable with the present model.

The failure to achieve Bloom-Gilman duality shows that the present version of the model is not advanced enough to handle this subtle question. Indeed, the lack of BloomGilman duality teaches us an important lesson. One might use either hadronic or quark degrees of freedom according to which leads to the simpler description of the specific problem at hand. Since both sets of states are complete, it is natural to expect that BloomGilman duality should result. The present work shows that such a supposition is not correct. Instead, the observed validity of both global and local forms of duality for deep inelastic scattering must be related to a deeper feature of QCD.

Although the present model is very simple, it suggests a prediction that if deep inelastic scattering experiments were to be made on the pion, Bloom-Gilman duality would not be observed. This is because of the quark-antiquark structure of the valence wave function that generally leads to a $1 / Q^{2}$ behavior of form factors at high momentum transfer.

Bloom and Gilman understood their duality in terms of the asymptotic fall of resonance transition form factors, quark counting rules and a simple scaling function to represent the high $Q^{2}$ data. The finer details of this analysis have not withstood the test of time, but measurements have shown that their duality still is viable. The underlying origin of this phenomenon is deeply buried within the confinement aspects of QCD. Its ultimate understanding remains a mystery.

\section{ACKNOWLEDGMENTS}

This work was supported by the U.S. Department of Energy Office of Science, Office of Nuclear Physics under Award No. DE-FG02-97ER-41014 and by Battelle Memorial Institute, Pacific Northwest Division Acting Under Contract No. DE-AC05-76RL01830 with the U.S. Department of Energy. Thank you to Dr. Stanley Brodsky and Dr. Guy de Teramond for the useful discussions regarding this work.
[1] W. Melnitchouk, R. Ent, and C. Keppel, Quark-hadron duality in electron scattering, Phys. Rep. 406, 127 (2005).

[2] E. D. Bloom and F. J. Gilman, Scaling, Duality, and the Behavior of Resonances in Inelastic electron-Proton Scattering, Phys. Rev. Lett. 25, 1140 (1970).

[3] E. D. Bloom and F. J. Gilman, Scaling and the behavior of nucleon resonances in inelastic electron-nucleon scattering, Phys. Rev. D 4, 2901 (1971).

[4] A. De Rujula, H. Georgi, and H. D. Politzer, An explanation of local duality and precocious scaling, Phys. Lett. B 64, 428 (1976).
[5] A. De Rujula, H. Georgi, and H. D. Politzer, Demythification of electroproduction, local duality and precocious scaling, Ann. Phys. (N.Y.) 103, 315 (1977).

[6] I. Niculescu et al., Experimental Verification of Quark Hadron Duality, Phys. Rev. Lett. 85, 1186 (2000).

[7] I. Niculescu et al., Evidence for Valencelike Quark Hadron Duality, Phys. Rev. Lett. 85, 1182 (2000).

[8] R. Ent, C. E. Keppel, and I. Niculescu, Nucleon elastic form-factors and local duality, Phys. Rev. D 62, 073008 (2000). 
[9] M. B. Einhorn, Confinement, form factors, and deepinelastic scattering in two-dimensional quantum chromodynamics, Phys. Rev. D 14, 3451 (1976).

[10] G. Domokos, S. Kovesi-Domokos, and E. Schonberg, Direct-channel resonance model of deep-inelastic electron scattering. I. scattering on unpolarized targets, Phys. Rev. D 3, 1184 (1971).

[11] G. Domokos, S. Kovesi-Domokos, and E. Schonberg, Deep-inelastic neutrino scattering in a resonance model, Phys. Rev. D 4, 2115 (1971).

[12] N. Isgur, S. Jeschonnek, W. Melnitchouk, and J. W. Van Orden, Quark hadron duality in structure functions, Phys. Rev. D 64, 054005 (2001).

[13] S. Jeschonnek and J. W. Van Orden, Modeling quark hadron duality for relativistic, confined fermions, Phys. Rev. D 69 , 054006 (2004).

[14] F. E. Close and Q. Zhao, A Pedagogic model for deeply virtual Compton scattering with quark hadron duality, Phys. Rev. D 66, 054001 (2002).

[15] D. R. Harrington, Asymptotic freedom for nonrelativistic confinement, Phys. Rev. C 66, 065205 (2002).

[16] F. E. Close and N. Isgur, The Origins of quark hadron duality: How does the square of the sum become the sum of the squares?, Phys. Lett. B 509, 81 (2001).

[17] M. W. Paris and V. R. Pandharipande, Scaling of space and time-like response of confined relativistic particles, Phys. Lett. B 514, 361 (2001).

[18] M. W. Paris and V. R. Pandharipande, Final state interaction contribution to the response of confined relativistic particles, Phys. Rev. C 65, 035203 (2002).

[19] S. J. Brodsky, G. F. de Teramond, H. G. Dosch, and J. Erlich, Light-front holographic QCD and emerging confinement, Phys. Rep. 584, 1 (2015).

[20] S. J. Brodsky, H.-C. Pauli, and S. S. Pinsky, Quantum chromodynamics and other field theories on the light cone, Phys. Rep. 301, 299 (1998).

[21] P. A. M. Dirac, Forms of relativistic dynamics, Rev. Mod. Phys. 21, 392 (1949).

[22] G. F. de Teramond and S. J. Brodsky, Light-Front Holography: A First Approximation to QCD, Phys. Rev. Lett. 102, 081601 (2009).

[23] S. J. Brodsky and G. F. de Teramond, Hadronic Spectra and Light-Front Wavefunctions in Holographic QCD, Phys. Rev. Lett. 96, 201601 (2006).

[24] S. J. Brodsky and G. F. de Teramond, Light-front dynamics and AdS/QCD correspondence: The pion form factor in the space- and time-like regions, Phys. Rev. D 77, 056007 (2008).

[25] J. Polchinski and M. J. Strassler, Deep inelastic scattering and gauge / string duality, J. High Energy Phys. 05 (2003) 012 .
[26] S. D. Drell and T.-M. Yan, Connection of Elastic Electromagnetic Nucleon Form-Factors at Large Q**2 and Deep Inelastic Structure Functions Near Threshold, Phys. Rev. Lett. 24, 181 (1970).

[27] G. B. West, Phenomenological Model for the Electromagnetic Structure of the Proton, Phys. Rev. Lett. 24, 1206 (1970).

[28] S. J. Brodsky and G. F. de Teramond, Light-front dynamics and AdS/QCD correspondence: Gravitational form factors of composite hadrons, Phys. Rev. D 78, 025032 (2008).

[29] Z. Abidin and C. E. Carlson, Gravitational form factors of vector mesons in an AdS/QCD model, Phys. Rev. D 77, 095007 (2008).

[30] S. D. Drell and T.-M. Yan, Connection of Elastic Electromagnetic Nucleon form Factors at Large $Q^{2}$ and Deep Inelastic Structure Functions Near Threshold, Phys. Rev. Lett. 24, 181 (1970).

[31] A. Karch, E. Katz, D. T. Son, and M. A. Stephanov, Linear confinement and AdS/QCD, Phys. Rev. D 74, 015005 (2006).

[32] G. F. de Téramond and S. J. Brodsky, Light-Front Holography: A First Approximation to QCD, Phys. Rev. Lett. 102, 081601 (2009).

[33] G. F. de Téramond, H. G. Dosch, and S. J. Brodsky, Baryon spectrum from superconformal quantum mechanics and its light-front holographic embedding, Phys. Rev. D 91, 045040 (2015).

[34] H. G. Dosch, G. F. de Téramond, and S. J. Brodsky, Superconformal baryon-meson symmetry and light-front holographic QCD, Phys. Rev. D 91, 085016 (2015).

[35] G. P. Lepage and S. J. Brodsky, Exclusive processes in perturbative quantum chromodynamics, Phys. Rev. D 22, 2157 (1980).

[36] Y. Li, P. Maris, X. Zhao, and J. P. Vary, Heavy quarkonium in a holographic basis, Phys. Lett. B 758, 118 (2016).

[37] G. A. Miller and S. J. Brodsky, Frame-independent spatial coordinate $\tilde{z}$ : Implications for light-front wave functions, deep inelastic scattering, light-front holography, and lattice QCD calculations, Phys. Rev. C 102, 022201 (2020).

[38] R. P. Feynman, Photon-Hadron Interactions (W.A. Benjamin, Inc., Reading, 1972).

[39] S. J. Brodsky, M. Burkardt, and I. Schmidt, Perturbative QCD constraints on the shape of polarized quark and gluon distributions, Nucl. Phys. B441, 197 (1995).

[40] L. L. Frankfurt, G. A. Miller, and M. Strikman, The Geometrical color optics of coherent high-energy processes, Annu. Rev. Nucl. Part. Sci. 44, 501 (1994).

[41] L. Frankfurt, G. A. Miller, and M. Strikman, Precocious dominance of point-like configurations in hadronic formfactors, Nucl. Phys. A555, 752 (1993). 